\title{
COARCTATION OF THE AORTA WITH CEREBRAL ANEURYSM
}

\author{
BY \\ J. N. O'REILLY, D.M., M.R.C.P. \\ Medical Superintendent of St. Margaret's Emergency Hospital, Epping
}

AND

\section{O. W. CHAPMAN, M.B., Medical Registrar to the Hospital}

Coarctation of the aorta is a rare condition. Death is due to rupture of cerebral aneurysm in so small a percentage that the following case seems worth reporting:

A boy, C. L., aged thirteen years, was admitted to hospital on March 3, 1941, with the history that four days previously while playing he had had a sudden general constricting sensation in the head. He had gone home and there vomited. The constricting sensation localized to a severe frontal headache which had continued. There had been periodical vomiting and the bowels were constipated. Prior to admission he had been given an enema during which he had collapsed, and his doctor had reported that he had had a fit. On admission the boy was semi-conscious, the pupils were equal and regular and reacted to light and accommodation. The cranial nerves were intact. There was considerable neck rigidity and Kernig's test was positive. The left knee jerk was present but the right knee jerk and both ankle jerks were absent. The biceps jerks were present and normal. The supinator jerks were exaggerated. The left plantar reflex was flexor but the right was doubtful. Examination of the fundi showed papilloedema. Sensation, movement and power in the limbs appeared normal. The cardio-vascular system showed some enlargement of the heart with the apex beat just outside the nipple line. The rhythm was regular. There was a rough systolic murmur in the precordium, best heard in the third left interspace. Blood pressure 230/140 $\mathrm{mm}$. Hg. No other marked physical signs.

Lumbar puncture showed increased pressure of the cerebrospinal fluid, about $28 \mathrm{~cm}$. of water, and marked blood-staining homogeneously distributed through the fluid. Examination showed 75,000 red blood cells per c.mm., 60 white blood cells per c.mm., protein 0.09 per cent., no other abnormality, and culture sterile.

During the forty-eight hours after admission the patient's condition improved, he became quite conscious and mentally normal, and on March 6 the headache was slight, blood pressure 180/120 mm. $\mathrm{Hg}$ and lumbar puncture showed the fluid straw coloured, still under increased pressure and sterile. The only additional clinical sign was that the right pupil was considerably larger than the left.

On March 9 the patient's headache was again severe and he was noticeably less alert. Plantar responses were extensor. Lumbar puncture showed deeply blood-stained fluid under $18 \mathrm{~cm}$. pressure.
Temperature was up to $101^{\circ} \mathrm{F}$. This condition continued during the next three days. By March 12 the headache was less and the cerebrospinal fluid was yellow and showed no evidence of fresh bleeding.

Full examination at this time revealed that the femoral arteries could not be palpated. The blood pressure in the popliteal artery was $105 / 100 \mathrm{~mm}$. $\mathrm{Hg}$ (the diastolic figure is somewhat doubtful). On these findings a diagnosis of coarctation of the aorta was made. An x-ray examination of the heart was made about this time and showed absence of the aortic knuckle which confirmed the diagnosis. The boy's general condition remained fairly satisfactory with temperature swinging up to about $100^{\circ} \mathrm{F}$. until March 20. On this day he complained of headache and he vomited. Later he became unconscious and lumbar puncture showed heavily blood-stained fluid. The next day he was still unconscious, the fluid was still deeply blood-stained and the temperature rose to $107^{\circ} \mathrm{F}$. Tepid sponging brought it down to $103^{\circ} \mathrm{F}$., but it rapidly returned to $107^{\circ} \mathrm{F}$. and within a few hours the patient died.

Post-mortem. On removing the sternum the heart was seen to be somewhat enlarged, and there was considerable dilatation of the aorta above the insertion of the ligamentum arteriosum. Below this the thoracic aorta quickly resumed its normal size and relations. On opening the heart the aortic valve was found to have two cusps only, one of apparently normal size and the other much enlarged and having a median raphe. The ascending and transverse aorta appeared normal on opening apart from the dilatation, but on passing a probe a complete obstruction was found at the insertion of the ligamentum arteriosum (fig. 1). The ligamentum itself was patent on the pulmonary side for about half its length and then became a solid cord. The left subclavian artery and the left thyroid axis were dilated. There was gross enlargement of the internal mammary arteries which could be followed down quite easily to their anastomoses with the deep epigastric vessels. The descending aorta tapered rapidly and the iliac vessels were small. On opening the cranium a large mass of blood clot was found lying at the base of the brain and tracking down into the spinal canal. This was found to be coming from a ruptured aneurysm of the anterior communicating artery at its junction with the left anterior cerebral artery (fig. 2). The blood had torn up the brain substance to some extent in both frontal lobes and it had ruptured into the anterior horn of the left 
lateral ventricle (fig. 3). There was another small

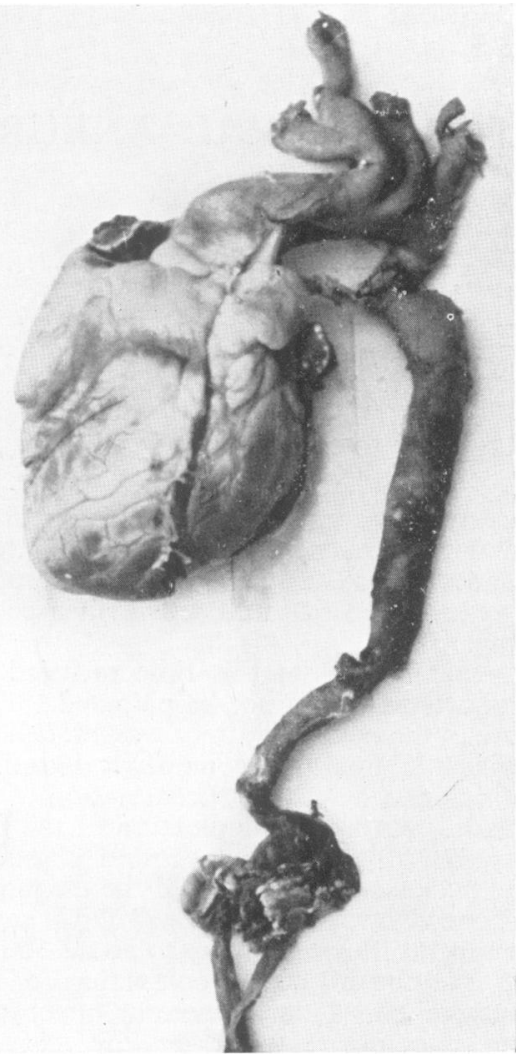

Fig. 1. - Heart and aorta showing constriction at insertion of ligamentum arteriosum. aneurysm on the left choroidal artery. No other abnormalities were found.

\section{Discussion}

Coarctation of the aorta is a well-known but by no means common abnormality.

Abbott (1928), quoting Paul White's figures, gives the frequency as two in five thousand consecutive autopsies. Blackford (1928) reports it as occurring once in 1588 consecutive autopsies. Other authors give similar figures. Turning to the clinical cases described, Blackford enumerates seventy-eight cases which he found in the literature, but of these only four were in children. Between 1928 and 1938 Eisenberg (1938) found about forty cases had been described in adults between seventeen and sixty-six

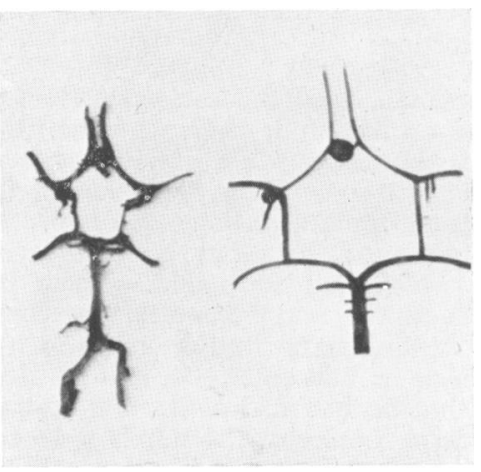

FIG. 2.-Circle of Willis, showing aneurysms: diagram alongside.

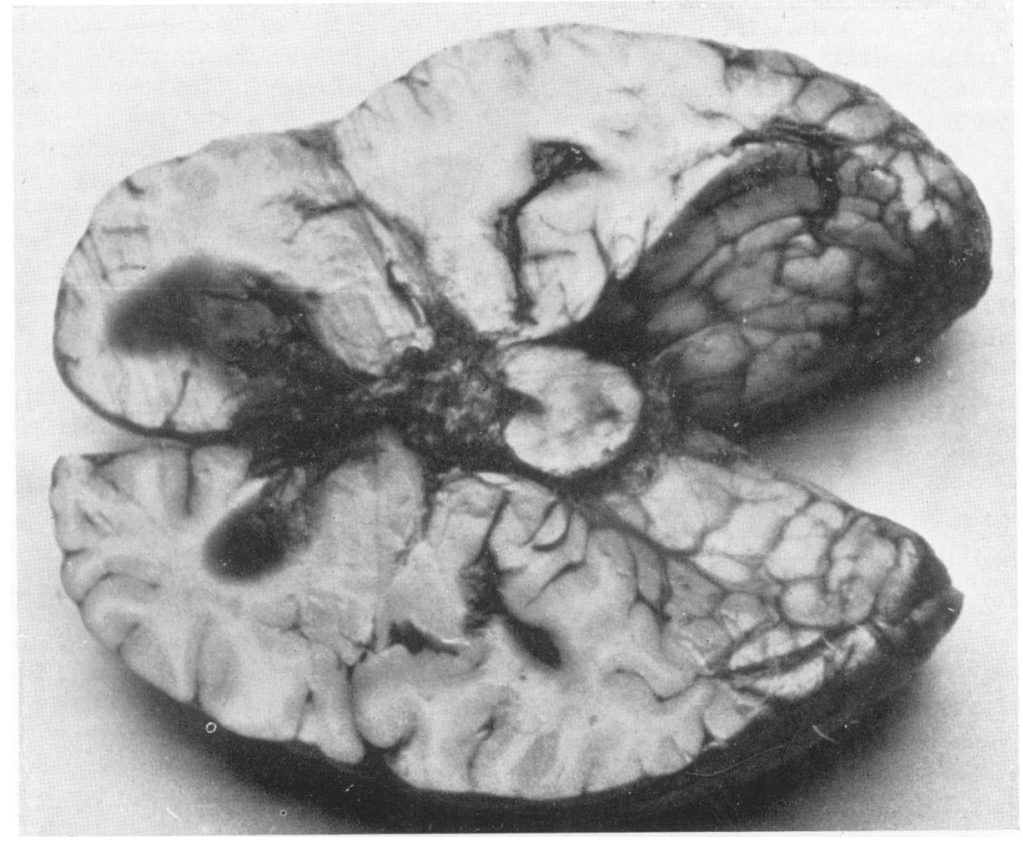

FIG. 3.-Section of brain, showing bloodclot in the lateral ventricle. 
years, whereas only eleven cases are recorded as occurring in children. To the latter he adds three of his own, two discovered in the course of routine examination and one which attended his cardiac clinic complaining of pain in the chest and legs on walking. He calls attention to the comparative rarity with which coarctation is diagnosed in children and says that palpation of the femoral pulses should form part of the routine examination of every child.

The diagnostic point is the contrast between the forceful bounding pulse and the high blood pressure in the arms with the feeble or absent pulse and lower pressure in the legs. A systolic murmur is also usually audible along the left border of the sternum. Evidence of collateral circulation around the scapula may be absent or unconvincing in children. Radiologically there is absence of the aortic knob in the postero-anterior view and a defect in the aortic arch in the left oblique view (Fray, 1930). Fray also describes the notching of the ribs seen in the $x$-ray film, an appearance first described at autopsy by Meckel.

The location of the stenosis is at the insertion of the ductus arteriosus in fifty per cent. of cases (Abbott), and just above or just below in the remaining fifty per cent. The ductus is commonly closed, either ligamentous throughout or in a few cases open on the pulmonary side (Fletcher, 1842).

The degree of stenosis is variable. Out of two hundred cases Abbott found it complete in fortyseven. Associated abnormalities of considerable degree are not common, with one exception, that of bicuspid aortic valve which appears in about twentyfive per cent. of the cases. This condition is a true ante-ratal abnormality due to fusion of the two cusps, and it is not uncommon to find, as in the present case, a median raphe either completely dividing the cusp into two, or itself fenestrated and only partially dividing the composite cusp.

The mode of death is usually decompensation or by rupture of the aorta. Mycotic aneurysm due to bacterial endocarditis is also frequent. A certain number of deaths are due to cerebral haemorrhage. In Abbott's series thirteen cases had 'spontaneous' rupture and five deaths were due to ruptured cerebral aneurysm. It is possible that in some of the other thirteen cases death was in fact due to rupture of an aneurysm.

Cerebral aneurysm is not a common condition, but it is one which has been described by a number of authors.

Eppinger first suggested it as a cause of cerebral haemorrhage in healthy young adults as long ago as 1887. Since then it has been described on a number of occasions, notably by Gowers (1893), Symonds (1923) and Parker (1926). Parker groups the aneurysms anatomically, giving the middle cerebral artery as the commonest site, 29 per cent. of a series of 154 cases. He quotes Gowers for the figure of eight aneurysms of the anterior communicating artery out of the same series $(5 \cdot 3$ per cent.).

The principal symptoms of aneurysm in the anterior portion of the cranium are severe neuralgic pain over the forehead and hyperaesthesia with loss of the corneal reflex. There may be paralysis of the third, fourth and sixth cranial nerves and exophthalmos. Sometimes there is a central scotoma with pallor of the optic disc on the side of the aneurysm and mild signs of pyramidal disturbances on the opposite side. If the aneurysm leaks there is increase of the focal signs, confusion or stupor, the temperature is raised up to about $101^{\circ} \mathrm{F}$.; the cerebrospinal fluid is blood-stained and under pressure. Parker describes a case of leak and finally rupture of a cerebral aneurysm in a healthy young man who was found also to have coarctation. The lesion was at the junction of the posterior communicating artery and the middle cerebral, and another aneurysm was found at the junction of the anterior communicating with the anterior cerebral artery. More recently Walker and Livingstone (1938) have described a case of coarctation which died following rupture of an aneurysm of the left vertebral artery within the cranium. The cause of aneurysm is attributed by Green (1928) and Forbus (1930) to a herniation of the intima of an artery at its junction with another artery. They have both shown that the muscular coat is deficient at this point and hernia is brought about by the constant high pressure due to the coarctation.

\section{Summary}

A case of recurrent cerebral haemorrhage occurring in a boy of thirteen years is described. The case was diagnosed as being due to leakage from a cerebral aneurysm associated with coarctation of the aorta, and this was confirmed at post-mortem.

Thanks are due to Dr. W. A. Bullough, County Medical Officer of Essex, for permission to publish this case.

\section{REFERENCES}

Abbott, M. (1928). Amer. Heart J., 3, 574.

Blackford, L. M. (1928). Arch. intern. Med., 41, 702.

Eisenberg, G. (1938). J. Pediat., 13, 303.

Eppinger, L. (1887). Arch. Klin. Chir. 35, 1.

Fletcher, T. B. (1842). Med.-chir. Trans., 25, 282.

Forbus, W. D. (1930). Johns Hopk. Hosp. Bull., 47, 239.

Fray, W. W. (1930). Amer. J. Roentgenol., 24, 349.

Gowers, W. R. (1893). A manual of diseases of the nervous system, second edition, Lond., 2.

Green, F. H. K. (1928). Quart. J. Med., 21, 419.

Parker, H. L. (1926). Arch. Neurol. Psychiat. Lond., 16, 728 .

Symonds, C. P. (1923). Guy's Hosp. Rep.,73, 159.

Walker, J. B., and Livingstone, F. D. M. (1938). Lancet, 2,660 . 